\title{
Multifocal visual evoked potential for evaluation of open-angle glaucoma
}

Mostafa EmadEldeen Hussien Mohamed Afify ${ }^{1}$, Randa Hesham Ali Abdelgawad ${ }^{1}$, Momen

Mahmoud Hamdi ${ }^{1}$, Amany Abd El-Fattah El-Shazly ${ }^{1}$ and Mohamed Adel Abdelshafik ${ }^{1}$

${ }^{1}$ Ophthalmology Department, Ain Shams University Hospitals, Cairo, Egypt

\begin{abstract}
Background: To correlate multifocal visual evoked potential (mfVEP) findings with static automated perimetry (SAP) and spectral-domain optical coherence tomography (SD-OCT) in eyes with primary openangle glaucoma (POAG).

Methods: This cross-sectional study included a consecutive sample of 40 eyes of 40 patients with POAG. The participants underwent a complete ophthalmologic assessment, axial length (AL) measurement, and assessments with SAP, SD-OCT, and mfVEP.

Results: POAG cases were aged $49.70 \pm 14.16$ years (mean \pm SD) and most were females $(\mathrm{n}=24,60 \%)$. For eyes of patients with POAG, the mfVEP upper-ring signal-to-noise ratio (SNR) showed a significant negative correlation with best-corrected $\log$ MAR visual acuity $(\mathrm{r}=-0.33 ; P=0.038)$, and a significant positive correlation with the superior hemifield of the visual field (VF) and the inferior-quadrant retinal nerve fiber layer $(\mathrm{RNFL})$ thickness $(\mathrm{r}=+0.34 ; P=0.030 ; \mathrm{r}=+0.51 ; P<0.001$, respectively). Similarly, the $\mathrm{mfVEP}$ lower-ring SNR showed a significant negative correlation with best-corrected logMAR visual acuity $(\mathrm{r}=-0.36 ; P=0.024)$ and a significant positive correlation with the inferior hemifield of the VF and superior quadrant RNFL thickness ( $\mathrm{r}=+0.55 ; P<0.001$ and $\mathrm{r}=+0.70 ; P<0.001$, respectively).

Conclusions: $\mathrm{mfVEP}$ is a promising tool for objective assessment of the VF in patients with POAG, as it is positively correlated with the VF and OCT RNFL thickness. Future longitudinal studies with a larger sample size and a specific glaucoma subtype, along with multiple follow-up evaluations, are warranted to confirm our preliminary results.
\end{abstract}

\section{KEYWORDS}

multifocal visual evoked potential, mfVEP, static automated perimetry, spectral-domain optical coherence tomography, SD-OCT, visual field, POAG, primary open-angle glaucoma, retinal nerve fiber layer thickness, best-corrected visual acuity, logarithm of the minimum angle of resolution

\section{INTRODUCTION}

Over 44 million patients have primary open-angle glaucoma (POAG), one of the most common causes of blindness worldwide [1]. POAG damage is usually assessed by optic disc photography, retinal nerve fiber layer (RNFL) assessment, and visual field (VF) examination [2].

Correspondence: Mostafa EmadEldeen Hussien Mohamed Afify, Assistant Lecturer of Ophthalmology, Faculty of Medicine, Ain Shams University, 12-Zahraa Second Stage, Tenth District, Nasr City, Cairo, Egypt. Email: mostafa.emad.afifi@gmail.com. ORCHID iD: https://orcid.org/0000-0002-0251-7092

How to cite this article: Afify MEHM, Abdelgawad RHA, Hamdi MM, El-Shazly AAE, Abdelshafik MA. Multifocal visual evoked potential for evaluation of open-angle glaucoma. Med Hypothesis Discov Innov Ophthalmol. 2021 Fall; 10(3): 114-120. https://doi.org/10.51329/mehdiophthal1429

Received: 26 August 2021; Accepted: 29 September 2021

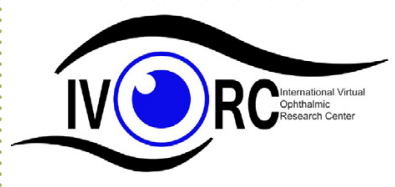

Copyright (C) Author(s). This is an open-access article distributed under the terms of the Creative Commons Attribution-NonCommercial 4.0 International License (http://creativecommons.org/licenses/by-nc/4.0/) which permits copy and redistribute the material just in noncommercial usages, provided the original work is properly cited. (c) (i) (s) 
Glaucomatous damage presents late symptoms. Early detection of this damage is necessary for earlier diagnosis and better prognosis. However, estimating an accurate diagnosis before the loss of function becomes marked is challenging. Once VF deficits are detected, $20 \%$ of retinal ganglion cells (RGCs) are already devastated by the disease [3]. As a subjective test, static automated perimetry (SAP) results are notably affected when patient cooperation during the test is poor [4].

Globally, optical coherence tomography (OCT) and SAP are commonly used for POAG management. OCT is able to show the RNFL and optic nerve head, and to measure the thicknesses accurately, making it a particularly important tool for the objective assessment of glaucomatous damage and progression [5]. Recently, introduction of spectral-domain OCT (SD-OCT) has revolutionized the imaging process of the RNFL, RGC layer, and inner plexiform layer, which are the principal sites of glaucomatous damage [6].

Multifocal visual evoked potential (mfVEP) is a new objective tool that records the cortically evolved electrical responses to a checkerboard patterned stimulus. mfVEP tests the central $22^{\circ}$ from the fixation point [7]. Recent studies have shown that mfVEP could provide a topographic assessment of VF defects of optic neuropathies, particularly the glaucomatous type, correlating well with automated perimetry results, and can predict SAP results in some cases [7].

This study aimed to investigate the correlation of mfVEP with SAP and RNFL thickness measured by OCT in eyes with POAG.

\section{METHODS}

This cross-sectional study was conducted between March 2018 and September 2020. The study was carried out on patients attending the ophthalmology outpatient clinic of Ain Shams University Hospitals, mainly Eldemerdash Medical Hospital, Cairo, Egypt. The study was conducted in accordance with the ethical standards of the Faculty of Medicine-Ain Shams University. The study protocol adhered to the principles of the Declaration of Helsinki and the institutional ethics committee approved the study protocol. Informed consent was obtained from all participants prior to enrollment in the study.

We included a consecutive sample of 40 eyes of 40 patients with POAG. POAG diagnosis was based on disc signs of glaucoma (lack of following the inferior $\geq$ superior $\geq$ nasal $\geq$ temporal neuroretinal rim thickness [ISNT] rule, thinning of the neural rim, disc hemorrhage, and inter-eye asymmetry of 0.2 or more in the vertical cup-to-disc ratio, with similar disc size), and field changes in glaucoma according to the Hodapp, Parrish, and Anderson's criteria [8]. Early glaucomatous defects were defined by a mean deviation (MD) less than -6 decibel $(\mathrm{dB})$, fewer than $25 \%$ of the points depressed below the $5 \%$ level, and fewer than 10 points depressed below the $1 \%$ level on the pattern deviation plot, and all points in the central $5^{\circ}$ had a sensitivity of $<$ $15 \mathrm{~dB}$. More advanced glaucoma was defined as moderate and severe glaucoma according to Hodapp, Parrish, and Anderson's criteria [8]. In the gonioscopy examination, all patients had a Shaffer angle grade III-IV.

The exclusion criteria were closed-angle glaucoma, ocular trauma, diabetes mellitus, and pre-existing posterior segment disease. We also excluded eyes with best-corrected distance visual acuity (BCDVA) less than 6/60 (logarithm of the minimum angle of resolution $[\log \mathrm{MAR}] \leq 1$ ) [9], errors of refraction $> \pm 3$ diopters [10], axial length $(\mathrm{AL})<23 \mathrm{~mm}$ and $>25 \mathrm{~mm}[11]$, as these factors may affect the results of $\mathrm{mfVEP}$ [12]. Because we wanted to examine the effect of a marked reduction of VF on the results of mfVEP, we did not exclude advanced stage POAG (MD $\geq-15 \mathrm{~dB})$.

All patients had a complete history and ophthalmic examination, including BCDVA using the Early Treatment Diabetic Retinopathy Study (ETDRS) visual acuity chart, and were recorded in both the logMAR notation and Snellen ratio. Ocular examinations were performed using a slit lamp BQ 900 (Haag-Streit AG, Koeniz, Switzerland), Goldmann applanation tonometry (Haag Streit, Koeniz, Switzerland), posterior segment examination using a $+90 \mathrm{D}$ lens, AL measurement using A-scan ultrasound (Pacscan $300 \mathrm{~A}$ A-scan ultrasound, Sonomed Inc., NY, USA), SAP using a Humphrey Field Analyzer (Carl Zeiss Meditec, Inc., Dublin, California, USA), OCT using a Nidek RS-3000 Advance Capture SD-OCT (Nidek, Gamagori, Japan; software version Navis-Ex 1.4.0.1), and mf VEP using the RETI system (Roland Consult, Brandenburg, Germany).

For the VF test, glaucomatous patients who had previous experience with field examinations and had a reliable test were accepted in the study. However, for patients new to the field examination, the first examination was used for familiarization, while the results of the second field test were included in the study. The field test was done using the 24-2 SITA-Standard VF test (24-2 Swedish interactive threshold algorithm standard, Humphrey Field Analyzer III; Carl Zeiss Meditec, Inc.), testing 54 points of Goldman size III (0.43 
degree) with a background luminance of 31.5 Apostilb (asb). A reliable SAP result was defined as fixation losses of $<20 \%$ and false-positive or false-negative responses of $<30 \%$ [13]. The same technician performed the test for all subjects, to limit inter-observer variation. The presence of VF defects in the superior or inferior hemifield detected on the pattern deviation plot was considered to check for possible correlation with the signal-to-noise ratio (SNR) of the corresponding superior or inferior mf VEG ring.

For SD-OCT imaging, a disc map protocol $(6 \times 6 \mathrm{~mm})$ was used to perform RNFL thickness measurement. A signal strength index $\geq 7 / 10$ was accepted as a reliable scan result. A scan density of $512 \mathrm{~A}$-scans (horizontal) $\times 128 \mathrm{~B}$-scans (vertical) was used to capture a detailed image of RNFL thickness in the peripapillary area. A 3.45-mm-diameter circle was centered around the optic disc, to estimate RNFL thickness in the four main quadrants (superior, inferior, nasal, and temporal). The same technician performed the test for all subjects, to limit inter-observer variation.

For the mfVEP, a 19-inch LCD monitor was used to present the stimulus for the Roland RETI system (Roland Consult, Brandenburg an der Havel, Germany). The pattern reversal dartboard stimulus was used to test the central $22^{\circ}$ of the VF. The stimulus was divided into sectors, and each sector contained eight black checks alternated with eight white checks, with each check reversing at a regular frequency in the pseudorandom m-sequence. The stimulus used a scaling factor of 1.4, relying on cortical magnification factors. The patient viewed the stimulus monocularly for four cycles, each lasting $2 \mathrm{~min}$ and $20 \mathrm{~s}$.

The process of recording $\mathrm{mfVEP}$ started with having accurate patient refraction in place, dilating the pupil, positioning the patient at a distance of $30 \mathrm{~cm}$ from the stimulus, and recording the monocular response. The patient had to fixate on a red cross in the middle of the stimulus, and fixation was monitored using a built-in video camera system. The recording process was performed in a single session and required 22-35 min per eye. At the start of the recording, a cross-shaped device holding four different electrodes was placed at the back of the head, with a distance of $3.5 \mathrm{~cm}$ above, $3 \mathrm{~cm}$ below, $4 \mathrm{~cm}$ to the right, and $4 \mathrm{~cm}$ to the left of the inion. This cross-shaped electrode-holding device ensured an equal pressure distribution by the electrodes. The parameters were set at a sampling frequency of 1020 hertz $(\mathrm{Hz})$, impedance level below 10 kiloohms $(\mathrm{k} \Omega)$, and band filter cut-off frequencies between 1 and $30 \mathrm{~Hz}$. Four runs were required for each eye to achieve a good SNR. The same technician performed the test for all subjects to ensure proper placement of electrodes to achieve a good SNR [14] and to limit inter-observer variation.

Responses in the mfVEP were displayed as follows. MATLAB-based software was used for the signal processing. The signal was recorded in two intervals, first, between 50 and $150 \mathrm{~ms}$, which was expected to be stimulus-related. The second interval was between 230 and $330 \mathrm{~ms}$, which was expected to show no response. SNRs were computed for the 58 stimulated fields and each of the four channels by dividing the signal in the first interval with that of the second interval [14]. In addition to measuring the SNR, the amplitude and latency of each of the 58 responses were recorded and displayed. The root mean square was calculated by measuring the amplitude of responses across time intervals between 0 and $500 \mathrm{~ms}$ for each of the 58 responses per eye [14].

The correlation between mfVEP, VF, and SD-OCT RNFL was calculated as follows: The mfVEP results were displayed in five concentric rings at $2.5^{\circ}, 5.5^{\circ}, 9.9^{\circ}, 15.6^{\circ}$, and $22^{\circ}$ around the fixation point. Each ring

A

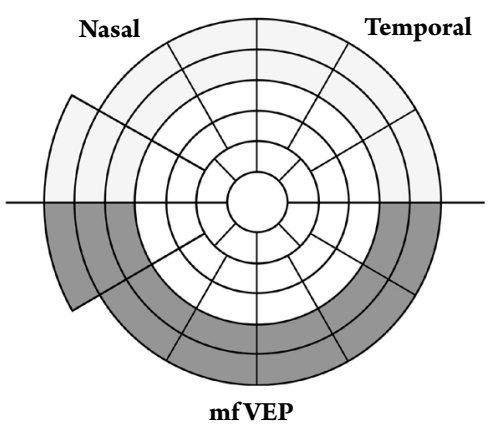

B

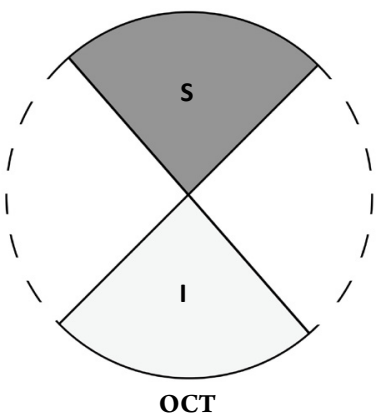

C

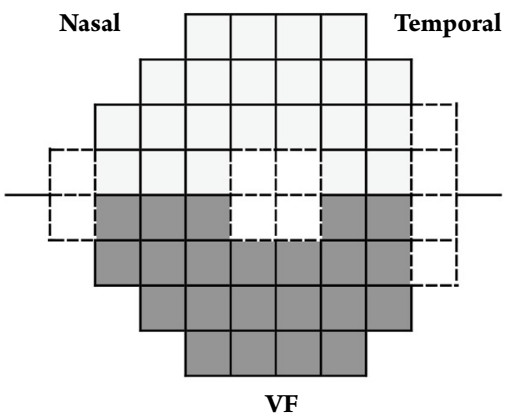

Figure 1. The upper-ring of the multifocal visual-evoked potential ( $\mathrm{mfVEP}$ ) is compared to that of the corresponding lower part of retinal nerve fiber layer (RNFL) thickness (I, inferior-quadrant) and area of the visual field (VF) marked in the superior hemifield. (A) mf VEP display chart with the upper half colored light grey, (B) corresponding to the lower quadrant of the RNFL measured using spectral-domain optical coherence tomography, and (C) the corresponding superior hemifield of the VF. 
was divided into segments, and the SNR of each segment was calculated. The SNR values of segments located between $9.9^{\circ}$ and $22^{\circ}$ were compared with the corresponding area of VF sensitivity and circumpapillary RNFL, as seen on an OCT volume disc scan (Figure 1). SD-OCT RNFL thickness analysis included lowerand upper-quadrants, only for correlation with the mfVEP upper-ring and mfVEP lower-ring [15, 16]. The outer two rings of the mfVEP display were included in analysis of the correlation with the corresponding VF hemifields, as this is the area the most commonly displays glaucomatous VF defects $[15,16]$.

The two most nasal points, four central points, and the most temporal four points of the 24-2 VF were excluded from the correlation analysis with the $\mathrm{mfVEP}$ display. The central four points of the VF corresponded to the inner three rings of the mf VEP display, occupying the central $10^{\circ}$ of the VF, as proposed by Hood and Greenstein $[15,16]$.

Data analysis was performed using SPSS Statistics for Windows (version 16.0; SPSS Inc., Chicago, IL, USA). The Kolmogorov-Smirnov test was used to verify the data normality assumption. The mean and standard deviation (SD) were used to describe quantitative data, whereas the numbers and percentages were used to describe qualitative factors. Correlation analyses were performed using Spearman's rho correlation statistics. Statistical significance was defined as a $P$-value of $<0.05$.

\section{RESULTS}

The mean \pm SD of the age of POAG cases was $49.70 \pm 14.16$ years with a male/female ratio of $16 / 24$. In terms of the severity of glaucoma, 19 patients showed mild damage, 10 patients showed moderate damage, and 11 patients showed severe damage. The demographic values and patient characteristics, such as age, level of intraocular pressure (IOP), BCDVA, AL, spherical equivalent (SE) in diopters, and cup-to-disc ratio are shown in Table 1.

As shown in Table 2, the mfVEP upper-ring SNR showed no significant correlation with age, SE, or AL ( $\mathrm{r}$ $=-0.19 ; P=0.230, \mathrm{r}=-0.07 ; P=0.646$, and $\mathrm{r}=-0.34 ; P=0.984$, respectively), yet there was a significant weak negative correlation with $\log$ MAR BCDVA and IOP $(r=-0.33 ; P=0.038$, and $r=-0.34 ; P=0.030$, respectively), a significant weak positive correlation with the superior hemifield of the $\operatorname{VF}(\mathrm{r}=+0.34 ; P=0.030)$, and a significant moderate positive correlation with the inferior-quadrant RNFL thickness $(\mathrm{r}=+0.51 ; P<0.001)$ (Table 2).

The mfVEP lower-ring SNR showed no significant correlation with age, SE, AL, and IOP $(r=-0.24$; $P=0.130, \mathrm{r}=-0.05 ; P=0.730, \mathrm{r}=-0.06 ; P=0.690$, and $\mathrm{r}=-0.14 ; P=0.370$, respectively). However, it showed a significantly weak negative correlation with $\log$ MAR BCDVA $(r=-0.36 ; P=0.024)$, a significant moderately positive correlation with the inferior hemifield of the VF $(\mathrm{r}=+0.55 ; P=<0.001)$, and a significantly strong positive correlation with the superior-quadrant RNFL thickness $(\mathrm{r}=+0.70 ; P<0.001)$ (Table 2).

Table 1. Baseline clinical and demographic characteristics of the study participants

\begin{tabular}{|l|l|}
\hline Variables & Value \\
\hline Age $(\mathbf{y})$, Mean \pm SD & $49.70 \pm 14.16$ \\
\hline Sex (Male/Female), $\mathbf{n}(\%)$ & $16(40) / 24(60)$ \\
\hline Laterality (OD/OS), n (\%) & $25(62.5) / 15(37.5)$ \\
\hline $\begin{array}{l}\text { Severity of POAG } \\
\text { Mild, n (\%) } \\
\text { Moderate, n (\%) } \\
\text { Sever, n (\%) }\end{array}$ & $19(47.5)$ \\
\hline BCDVA (Snellen), Mean \pm SD & $10(25)$ \\
\hline BCDVA (logMAR), Mean \pm SD & $11(27.5)$ \\
\hline SE (D), Mean \pm SD & $0.60 \pm 0.16$ \\
\hline IOP (mm Hg), Mean \pm SD & $0.20 \pm 0.16$ \\
\hline $\begin{array}{l}\text { AL (mm), Mean } \pm \text { SD } \\
\text { C/D ratio, Mean } \pm \text { SD }\end{array}$ & $-0.12 \pm 1.43$ \\
\hline $\begin{array}{l}\text { Abbreviations: y, years; SD, standard deviation; n, number; \%, percentage; OD, right eye; OS, left eye; POAG, primary open- } \\
\text { angle glaucoma; BCDVA, best-corrected distance visual acuity, Snellen, range of Snellen acuities ratios; logMAR, logarithm of the } \\
\text { minimum angle of resolution; SE, spherical equivalent; D, dioptre; IOP, intraocular pressure; mmHg, millimeter of mercury; AL, } \\
\text { axial length; mm, millimeter; C/D ratio, cup-to-disc ratio. }\end{array}$ \\
\hline
\end{tabular}


Table 2. Correlation between the mf VEP upper or lower-ring signal-to-noise ratio and each parameter in study participants

\begin{tabular}{|c|c|c|c|}
\hline \multicolumn{2}{|l|}{ Parameters } & \multirow{2}{*}{$\begin{array}{l}\text { mf VEP upper ring SNR } \\
-0.19\end{array}$} & \multirow{2}{*}{$\begin{array}{l}\text { mf VEP lower ring SNR } \\
-0.24\end{array}$} \\
\hline $\operatorname{Age}(y)$ & Correlation Coefficient & & \\
\hline & ${ }^{*} P$-value & 0.230 & 0.130 \\
\hline \multirow[t]{2}{*}{ SE (D) } & Correlation Coefficient & -0.07 & -0.05 \\
\hline & ${ }^{*} P$-value & 0.646 & 0.730 \\
\hline \multirow[t]{2}{*}{$\mathbf{A L}(\mathbf{m m})$} & Correlation Coefficient & -0.34 & -0.06 \\
\hline & ${ }^{*} P$-value & 0.984 & 0.690 \\
\hline \multirow[t]{2}{*}{ BCDVA (logMAR) } & Correlation Coefficient & -0.33 & -0.36 \\
\hline & ${ }^{*} P$-value & 0.038 & 0.024 \\
\hline \multirow[t]{2}{*}{ IOP $(\mathbf{m m H g})$} & Correlation Coefficient & -0.34 & -0.14 \\
\hline & ${ }^{*} P$-value & 0.030 & 0.370 \\
\hline \multirow{2}{*}{$\begin{array}{l}\text { VF, superior or inferior } \\
\text { hemifield }\end{array}$} & Correlation Coefficient & +0.34 & +0.55 \\
\hline & ${ }^{*} P$-value & 0.030 & $<0.001$ \\
\hline \multirow{2}{*}{$\begin{array}{l}\text { RNFLT, inferior or superior } \\
\text { quadrant }\end{array}$} & Correlation Coefficient & +0.51 & +0.70 \\
\hline & ${ }^{*} P$-value & $<0.001$ & $<0.001$ \\
\hline
\end{tabular}

Abbreviations: mfVEP, multifocal visual evoked potential; SNR, signal-to-noise ratio; y, years; SE, spherical equivalent; D, diopters; AL, axial length; mm, millimeters; BCDVA, best-corrected distance visual acuity; logMAR, the logarithm of the minimum angle of resolution; IOP, intraocular pressure; $\mathrm{mmHg}$, millimeter of mercury; VF, visual field; RNFLT, retinal nerve fiber layer thickness. ${ }^{*}$ Spearman correlation; $P$-value $<0.05$ is shown in bold.

\section{DISCUSSION}

Our study showed a significant positive correlation between the upper and lower-rings of the mfVEP and the corresponding hemifields of VF in patients with POAG. Similarly, the lower- and upper-quadrants of the RNFL showed a significant positive correlation with the upper and lower-rings of the mfVEP. The SNR of both the upper and lower-rings of mfVEP did not correlate with patients' age, in agreement with Nakamura et al. [11]. Likewise, the SNR of both the upper and lower-rings of mf VEP showed no correlation with the SE of patients, as expected, given that patients were appropriately refracted during the test. Because blurred vision due to either uncorrected refractive errors or cataracts could decrease the mfVEP responses according to Winn et al.s [17] and Nakamura et al.s [11] results, these shortcomings should be considered when performing mfVEP in clinical practice.

There was no statistically significant correlation between either the upper or lower-ring SNR of mf VEP with AL in patients with POAG, which agrees with the results of Nakamura et al. [11], who found that AL was not associated with the SNR-area under the curve and the central SNR-area under the curve. Additionally, high myopia significantly reduced the SNR-area under the curve of mfVEP responses, except in the central upper hemifield, despite refractive correction [11]. The logMAR BCDVA had a significant negative correlation with mfVEP upper- and lower-ring SNRs, which agreed with the results of Jiang et al. [18]. In their cross-sectional study of patients with macular diseases, they found that the mean $\mathrm{mfVEP}$ amplitude within a radius of the central $2^{\circ}, 5^{\circ}$, and $10^{\circ}$ of the VF of all affected eyes was significantly negatively correlated with the logMAR BCDVA. Despite having similar results to our study, the difference in the study populations and perimetry methods [18] may not allow a definitive conclusion in this regard. Further studies are necessary to discover possible correlations and agreements between this electrophysiological test and logMAR BCDVA in patients with glaucoma.

The mfVEP upper- and lower-ring SNRs had a significant positive correlation with the superior and inferior hemifield of the VF in our study, respectively. These findings agree with the results of Goldberg et al. [19], Hood et al. [20], Horn et al. [14], and Qiao et al. [21]. Goldberg et al. [19] used a new method of mf VEP recording, the ObjectiVision system, and identified scotomas in the majority of cases with glaucoma who had established VF defects on subjective testing. Moreover, a large number of fellow eyes of their subjects with recent normal VF revealed abnormal multifocal objective perimetry responses. The authors proposed the possibility of identifying glaucomatous VF defects earlier by using this method than by using conventional perimetry [19]. Hood et al. [20] found that the decrease in the signal portion of the mfVEP response was proportional to Humphrey VF loss [20]. Likewise, our results confirmed a significant positive correlation between SNR values and VF in glaucomatous eyes. Nevertheless, we did not compare the SNR changes with mean deviation values in the Humphrey VF test. Similar to our findings, Horn et al. [14] reported a significant correlation between VEP amplitudes and VF defects. However, in contrast to our participants, who were POAG cases, they included diverse types of glaucomatous eyes with VF defects, including POAG, normal-tension glaucoma, secondary open-angle 
glaucoma, pigmentary glaucoma, or pseudoexfoliation [14]. Qiao et al. [21] compared the sensitivity of mf VEP with those of SAP for detecting visual pathway abnormalities in patients with pituitary adenomas and of OCT for RNFL thickness. Good agreement was found between the SAP and mfVEP findings, and the correlation between the mfVEP and VF scores was significant [21]. Similarly, we found a significant correlation between SAP results and mfVEP in patients with POAG. However, Qiao et al. studied pituitary adenoma patients [21].

We found a significant positive correlation between mfVEP upper- or lower-ring SNR and inferior- or superiorquadrants of RNFL thickness, respectively. These findings agree with the results of Moschos et al. [22], Horn et al. [14], and Qiao et al. [21]. Similar to our results, Horn et al. [14] found a significant correlation between VEP amplitudes and RNFL thickness, and this was a linear relationship between local mf VEP responses and corresponding RNFL thickness. However, they included a diverse type of glaucomatous eyes [14], in contrast to our participants who were POAG cases only. Qiao et al. [21] found a good agreement between OCT RFNL thickness and mfVEP findings, and the correlation values between RNFL thickness and mfVEP score were significant in their study. In addition, our study revealed a significant correlation between OCT RFNL thickness and mfVEP SNR. Yet, the two studies had different study populations. In a similar study design, Moschos et al. [22] recruited 29 eyes of patients with POAG and found a statistically significant moderately positive correlation between mfVEP amplitude and RNFL thickness in glaucomatous eyes. In our participants with POAG, the mfVEP upper-ring SNR showed a significant moderately positive correlation with inferior quadrant RNFL thickness, and the mfVEP lower-ring SNR showed a significant strong positive correlation with superior quadrant RNFL thickness. These findings are consistent with those reported by Moschos et al. [22], who proposed the combined application of $\mathrm{mfVEP}$ and OCT as a useful approach for monitoring glaucoma progression, which may lead to more appropriate treatment of the disease.

In a pilot study on glaucomatous eyes, Jindal et al. [23] examined the possible improvement in mf VEP after lowering IOP. They found poor mf VEP test-reliability in terms of SNR in many cases showing the greatest VF improvement. The study revealed no significant correlation between IOP reduction and changes in mf VEP. They postulated that a larger trial may verify improvement in electrophysiological results in patients with glaucoma after significant IOP reduction [23]. Although the aim of our study differed from that of their study, we found no significant correlation between mfVEP lower-ring SNR and IOP level, but found a significant weak negative correlation with mfVEP upper-ring SNR. The difference between the findings of these studies may be due to their small sample size and different machines, as they utilized the Accumap ObjectiVision Opera version 2.0 for recording mfVEP [23]. At present, it is not possible to comment with certainty on the existence or non-existence of a correlation between IOP and electrophysiological results in patients with glaucoma. Future longitudinal studies with a more sophisticated case selection and study design may provide a more robust conclusion.

This study had some strengths. First, this study presented a new method for analyzing the display of mf VEP. Second, this study presented a new technique for accurate assessment of correlation between mfVEP, VF, and RNFL thickness in patients with POAG. However, our work was also limited in a few respects. First, the sample size was small. Second, follow-up was not performed to ensure reproducibility of the results. Third, there was an absence of a normative database in our device. Future studies are needed to validate the mf VEP in glaucomatous eyes, if this objective test is to be considered for assessing visual function in optic nerve diseases, including glaucomatous optic neuropathy. This can be achieved by measuring the sensitivity, specificity, positive-predictive value, and negative-predictive value. Furthermore, future studies with larger sample sizes are needed, with longer follow-up, to consolidate the value of the mfVEP in POAG management.

\section{CONCLUSIONS}

Assessing mf VEP is a promising tool for objective evaluation of VF, particularly in patients with POAG, as it showed a positive correlation with VF analysis and OCT-based RNFL thickness in these patients. However, future cohort studies with more sophisticated case selection may provide a more robust conclusion.

\section{ETHICAL DECLARATION}

Ethical approval: The study was conducted in accordance with the ethical standards of the Faculty of MedicineAin Shams University, Cairo, Egypt. The study protocol adhered to the principles of the Declaration of Helsinki and the institutional ethics committee approved the study protocol. Informed consent was obtained from all participants prior to enrollment in the study.

Conflict of interests: None. 


\section{FUNDING}

None.

\section{ACKNOWLED GMENTS}

None.

\section{REFERENCES}

1. Tham Y-C, Cheng C-Y. Associations between chronic systemic diseases and primary open angle glaucoma: an epidemiological perspective. Clinical \& Experimental Ophthalmology. 2017;45(1):24-32. doi: 10.1111/ceo.12763 pmid: 27083150

2. Gölemez H, Ylldırım N, Özer A. Is multifocal electroretinography an early predictor of glaucoma? Documenta Ophthalmologica. 2016;132(1):27-37. doi: 10.1007/s10633-016-9524-3 pmid: 26792427

3. Xu LJ, Zhang L, Li SL, Zemon V, Virgili G, Liang YB. Accuracy of isolated-check visual evoked potential technique for diagnosing primary open-angle glaucoma. Documenta Ophthalmologica. 2017;135(2):107-19. doi: 10.1007/s10633-017-9598-6 pmid: 28702796

4. Lucy KA, Wollstein G. Structural and functional evaluations for the early detection of glaucoma. Expert Review of Ophthalmology. 2016;11(5):367-76. doi: 10.1080/17469899.2016.1229599 pmid: 28603546

5. Ledolter AA, Monhart M, Schoetzau A, Todorova MG, Palmowski-Wolfe AM. Structural and functional changes in glaucoma: comparing the two-flash multifocal electroretinogram to optical coherence tomography and visual fields. Documenta Ophthalmologica. 2015;130(3):197-209. doi: 10.1007/s10633-015-9482-1 pmid: 25616700

6. Oddone F, Lucenteforte E, Michelessi M, Rizzo S, Donati S, Parravano M, et al. Macular versus Retinal Nerve Fiber Layer Parameters for Diagnosing Manifest Glaucoma. Ophthalmology. 2016;123(5):939-49. doi: 10.1016/j.ophtha.2015.12.041 pmid: 26891880

7. Young B, Eggenberger E, Kaufman D. Current electrophysiology in ophthalmology. Current Opinion in Ophthalmology. 2012;23(6):497-505. doi: 10.1097/ICU.0b013e328359045e pmid: 23047167

8. Brusini P, Johnson CA. Staging Functional Damage in Glaucoma: Review of Different Classification Methods. Survey of Ophthalmology. 2007;52(2):156-79. doi: 10.1016/j.survophthal.2006.12.008 pmid: 17355855

9. Jeon J, Oh S, Kyung S. Assessment of visual disability using visual evoked potentials. BMC Ophthalmology. 2012;12(1). doi: 10.1186/1471-2415-12-36 pmid: 22866948

10. Dani JS, Varlekar MD, Dani AS. Effects on visual evoked potential in myopia. Int J Basic Appl Physiol. 2020;9(1):41-46. Link

11. Nakamura M, Kato K, Kamata S, Ishikawa K, Nagai T. Effect of refractive errors on multifocal VEP responses and standard automated perimetry tests in a single population. Documenta Ophthalmologica. 2014;128(3):179-89. doi: 10.1007/s10633-014-9431-4 pmid: 24615593

12. Kothari R, Bokariya P, Singh R, Singh S. Influence of refractory error on the pattern reversal VEPs of myopes and hypermetropes. International Journal of Physiology. 2013;1(1):57-61. Link

13. De Moraes CG, Liebmann JM, Ritch R, Hood DC. Clinical use of multifocal visual-evoked potentials in a glaucoma practice: a prospective study. Documenta Ophthalmologica. 2012;125(1):1-9. doi: 10.1007/s10633-012-9324-3 pmid: 22476612

14. Horn FK, Kaltwasser C, Jünemann AG, Kremers J, Tornow RP. Objective perimetry using a four-channel multifocal VEP system: correlation with conventional perimetry and thickness of the retinal nerve fibre layer. British Journal of Ophthalmology. 2012;96(4):554-9. doi: 10.1136/bjophthalmol-2011-300844 pmid: 22116959

15. Hood D. Multifocal VEP and ganglion cell damage: applications and limitations for the study of glaucoma. Progress in Retinal and Eye Research. 2003;22(2):201-51. doi: 10.1016/s1350-9462(02)00061-7 pmid: 12604058

16. Hood DC, Anderson SC, Wall M, Kardon RH. Structure versus Function in Glaucoma: An Application of a Linear Model. Investigative Opthalmology \& Visual Science. 2007;48(8). doi: 10.1167/iovs.06-1401 pmid: 17652736

17. Winn BJ. Interpreting the multifocal visual evoked potential: the effects of refractive errors, cataracts, and fixation errors. British Journal of Ophthalmology. 2005;89(3):340-4. doi: 10.1136/bjo.2004.047910 pmid: 15722316

18. Jiang $\mathrm{L}$, Zhang $\mathrm{H}, \mathrm{Xie} J$ J, Jiao $\mathrm{X}$, Zhou H, Ji H, et al. Application of multifocal visual evoked potentials in the assessment of visual dysfunction in macular diseases. Eye. 2011;25(10):1302-9. doi: 10.1038/eye.2011.153 pmid: 21720415

19. Goldberg I, Graham SL, Klistorner AI. Multifocal objective perimetry in the detection of glaucomatous field loss 11 Drs Graham, and Klistorner have patents pending for techniques used by the ObjectiVision system and stock in ObjectiVision. Klistorner is a Sydney Medical Foundation research fellow. American Journal of Ophthalmology. 2002;133(1):29-39. doi: 10.1016/s0002-9394(01)012946 pmid: 11755837

20. Hood DC, Greenstein VC, Odel JG, Zhang X, Ritch R, Liebmann JM, et al. Visual Field Defects and Multifocal Visual Evoked Potentials: Evidence of a Linear Relationship. Archives of Ophthalmology. 2002;120(12):1672-81. doi: 10.1001/ archopht.120.12.1672 pmid: 12470141

21. Qiao N, Zhang Y, Ye Z, Shen M, Shou X, Wang Y, et al. Comparison of multifocal visual evoked potential, static automated perimetry, and optical coherence tomography findings for assessing visual pathways in patients with pituitary adenomas. Pituitary. 2015;18(5):598-603. doi: 10.1007/s11102-014-0613-6 pmid: 25349031

22. Moschos MM, Georgopoulos G, Chatziralli IP, Koutsandrea C. Multifocal VEP and OCT findings in patients with primary open angle glaucoma: A cross-sectional study. BMC Ophthalmology. 2012;12(1). doi: 10.1186/1471-2415-12-34 pmid: 22856337

23. Jindal AP, Fleischman D, Leiby B, Spaeth GL, Myers JS, Katz LJ. Effects of acutely lowering intraocular pressure on the results of multifocal visual evoked potential testing. Acta Ophthalmologica. 2011;89(7):e550-e4. doi: 10.1111/j.1755-3768.2011.02177.x pmid: 21599873 